Originales

\title{
Absentismo laboral por patología odontológica y médica en una industria de gran porte del sector mecánico y mueblero
}

\author{
Absenteeism by medical and dental pathologies in a large industry of the \\ mechanical and furniture field
}

Tânia Adas Saliba Rovida', Ronald Jefferson Martins', Artênio José Ísper Garbin', Cléa Adas Saliba Garbin'

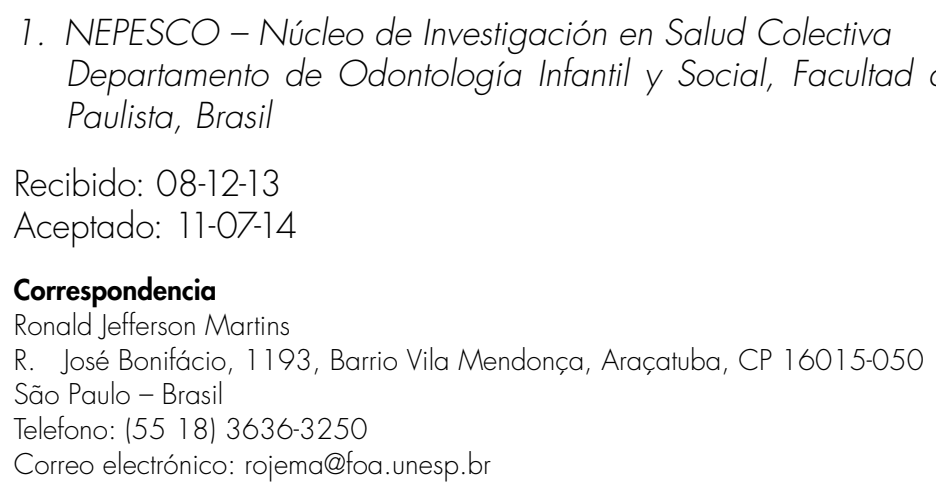

Resumen

Introducción: La legislación vigente brasileña establece que, en las industrias, los servicios de salud se realizan por el Servicio Especializado en Ingeniería de Seguridad y en Medicina del Trabajo (SESMT).

Objetivo: Analizar las causas del absentismo por razones odontológicas y médicas y verificar la interferencia de factores como edad, sexo y función del trabajador, además de razones que más llevaron al empleado a ausentarse de su trabajo.

Material y métodos: La investigación es un estudio descriptivo de abordaje cuantitativo. Se examinaron los certificados odontológicos y médicos y declaraciones de comparecencia debidamente homologados de una industria del sector acrílico del municipio de Araçatuba-SP, en el periodo de enero a julio de 2011.

Resultados: Del total de certificados $(\mathrm{n}=1841)$, solo $103(5,6 \%)$ presentaron razones odontológicas. Se observó predominancia de franja etaria de 20 a 29 años, sexo masculino y función no administrativa. Las causas odontológicas y médicas más comunes indicadas en los certificados que llevaron al funcionario a ausentarse del trabajo fueron «dientes incluidos e impactados» $\mathrm{y}$ "diarrea y gastroenteritis de origen infeccioso presumible", respectivamente. Sobre los factores relacionados al absentismo, se observó un índice de gravedad de 2,83, de frecuencia de 1,33 y la duración media de ausencias de 2,12.

Conclusiones: Se concluye que las razones odontológicas tuvieron poca importancia sobre el total de ausencias por motivos de enfermedad, además que provocaron el ausentismo del trabajador por un periodo menor. Las variables edad y función no influyeron en la ocurrencia del absentismo en el trabajo.

Med Segur Trab (Internet) 2014; 60 (236) 489-496

Palabras clave: Absentismo, Salud laboral, Salud bucal, Sector privado. 


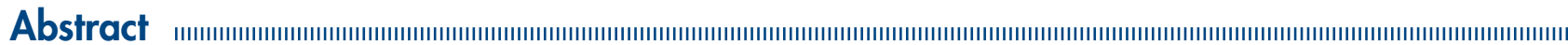

Introduction: The current Brazilian legislation states that health services must be performed by the Specialized Service of Safety Engineering and Labor Medicine (SESMT).

Objective: To analyze the dental and medical reasons for absenteeism, checking the interference of factors such as age, gender and position of the worker, and most of the reasons that led the employee to miss work.

Material and methods: The research appears as a descriptive study with a quantitative approach. The dental and medical certificates and statements of attendance were analyzed, duly approved, lodged in a acrylic industry in the city of Araçatuba-SP, from January to July 2011.

Results: Of the total number of certificates $(n=1841)$, only $103(5.6 \%)$ were for dental reasons. Predominance of the age group of 20-29 years, males and with non-administrative function. The reasons most dental and medical certificates stated that led the workers to miss working days were «inclusive and impacted teeth" and "diarrhea and gastroenteritis of presumed infectious origin", respectively. As to factors related to absenteeism, there was a severity index of 2.83, 1.33 frequency and average duration of absences of 2.12 .

Conclusions: It is concluded that dental reasons had little weight on total absences due to illness that caused the temporary leave of the worker for a shorter period. The age and function variables influence the occurrence of work absenteeism.

Med Segur Trab (Internet) 2014; 60 (236) 489-496

Key words: Absenteeism, occupational bealth, oral bealth, private sector. 


\section{INTRODUCCIÓN}

La seguridad y medicina del trabajo es el segmento del Derecho Laboral encargado de ofrecer condiciones de protección a la salud del trabajador en el local de trabajo; y de su recuperación cuando no se encuentre en condiciones de prestar servicios a su empleador ${ }^{1}$. En las industrias, los servicios de salud se realizan por el Servicio Especializado en Ingeniería de Seguridad y en Medicina del Trabajo (SESMT), por medio de la elaboración e implementación del Programa de Control Médico de Salud Ocupacional (PCMSO). Los profesionales que deberán componer el SESMT son los ingenieros de seguridad laboral, médico laboral, enfermero laboral, auxiliar de enfermería laboral y técnico de seguridad laboral. Como medidas preventivas de medicina laboral hay las consultas, orientaciones, ponencias en las empresas y los exámenes médicos obligatorios: los de ingreso, los ocupacionales periódicos y los de retiro ${ }^{1-3}$.

La implantación de servicios de salud específicos para grupos de trabajadores despierta intereses opuestos respecto al problema, pues la prioridad para el empresario es garantizar la presencia del trabajador en su puesto de trabajo y controlar el absentismo. Ya para los sindicatos es proteger la salud de los trabajadores ${ }^{4}$.

El absentismo laboral hace con que las empresas tengan que sustituir sus trabajadores ausentes, lleva a una disminución de la producción y de su calidad, mientras que los gastos de personal se mantienen iguales o superiores. El principal motivo del ausentismo al trabajo son problemas de salud, muchas veces generados por condiciones laborales ${ }^{4}$.

Hay dos tipos de absentismo: el tipo I es aquel representado por la ausencia simple del empleado al trabajo, de fácil mensuración y coste calculado, llevando a la pérdida de las horas no trabajadas. Ya el tipo II es el absentismo nombrado «cuerpo presente», cuando aunque el trabajador no falta al trabajo, no entrega su mejor desempeño, lo que lleva a la disminución en su productividad, además de predisponerlo a accidentes laborales ${ }^{5}$. La ocurrencia de ese tipo de absentismo es usual en las odontalgias, pues el dolor que empieza en la madrugada lleva a la persona a dormir mal y la impide de tener un sueño reparador. Al día siguiente, el individuo estará fatigado, irritado e incapaz de concentrarse ${ }^{6}$.

Aunque la especialidad Odontología Laboral haya sido reconocida por la Resolución CFO-25/2002, de 16 de mayo de $2002^{7}$, hasta el momento no existe referencia legal cualquiera sobre el Cirujano Dentista Laboral dentro del equipo del SESMT ${ }^{8}$. En realidad, muchos médicos actúan de buena gana al extender el examen de ingreso de un candidato hasta los dientes y, al encontrar raíces residuales, exigen o recomiendan su eliminación?

La inclusión de la Odontología en el PCMSO, igualmente que certifica la salud bucal dentro del sistema de salud ocupacional, crearía un banco de datos para el área de la Odontología. En el examen clínico de rutina y obligatorio del PCMSO, se podría incluir el examen odontológico, utilizándose inicialmente una ficha simplificada de la Organización Mundial de la Salud (OMS), en la que constan exámenes de dientes, mucosas y articulaciones, con la posibilidad de uso del Código Internacional de Enfermedades $(\mathrm{CIE})^{10,11}$.

El análisis de la distribución del absentismo conforme la edad, sexo y ocupación o cargo de los trabajadores es fundamental para orientar la toma de decisiones de las diferentes estrategias para su prevención ${ }^{4,12}$. Basándose en todo lo expuesto, se objetivó evaluar el absentismo por razones odontológicas y médicas, verificando la interferencia de factores como edad, sexo y función del trabajador en su ocurrencia.

\section{MATERIAL Y MÉTODOS}

La investigación es un estudio descriptivo, de abordaje cuantitativo. La industria estudiada fabricaba, entre otros productos, lavadoras de ropa y muebles metálicos, y su grado de riesgo es $3^{2,8}$. El grado de riesgo se define por la Norma Reguladora 4 (NR 4) y 
sirve para mensurar el riesgo de cada actividad laboral. Esa medida se determina al considerar el ramo de actividad de la empresa. El estudio se realizó en la ciudad de Araçatuba, Estado de São Paulo (Brasil).

Inicialmente, el investigador se dirigió al Departamento de Personal de la industria con el objetivo de aclarar la finalidad de la investigación y posterior uso de los datos recogidos. Así, se obtuvo el consentimiento de los directores de la empresa para acceso a los certificados odontológicos y médicos y al listado con el registro de los empleados.

Se analizaron todos los certificados y declaraciones de comparecencia debidamente homologados y emitidos en el periodo de enero a julio de 2012 y que sirvieron para justificar la ausencia del trabajador. De esos certificados, se recogieron datos referentes a la fecha, duración y motivo de la ausencia, basados en el Código Internacional de Enfermedades $(\mathrm{CIE}-10)^{11}$. De los registros de los trabajadores se analizaron informaciones como sexo, edad y función.

Para el cálculo del absentismo, se siguió la orientación del Subcomité de Absentismo de la Comisión Internacional de Salud Ocupacional, que indica como mínimo la obtención de dos índices esenciales: frecuencia, que es la relación entre el número de episodios de ausencia y el número de trabajadores; y gravedad, que es la relación entre el número de días de ausencia y el número de trabajadores. Por su importancia, se calculó también la duración media de las ausencias, obtenida por la relación entre el número de días de ausencia y el número de episodios de ausencia ${ }^{13}$. Se elaboró una base de datos en el programa Epi Info, versión 3.5.2, cuyos datos se analizaron estadísticamente por medio de análisis cuantitativo (comparación entre proporciones) y se presentaron en frecuencias absolutas y porcentuales. Se obtuvo el parecer favorable del Comité de Ética en Investigación en Seres Humanos de la Facultad de Odontología de Araçatuba-UNESP, Proceso FOA-01140/2011.

\section{RESULTADOS}

Durante el periodo de investigación la industria tenía 1380 empleados, de los que 275 (19,9\%) del sexo femenino y $1105(80,1 \%)$ del sexo masculino. Según la clasificación hecha por el Servicio Brasileño de Apoyo a las Micro y Pequeñas Empresas (SEBRAE) ${ }^{14}$, cuyo criterio de clasificación del porte de una empresa es el número de empleados, se trata de una gran empresa (más de 500 empleados).

Se analizaron 1841 certificados y declaraciones de comparecencia, 103 (5,6\%) odontológicos y 1738 (94,4\%) médicos. En total, 316 (17,2\%) eran de mujeres y 1525 $(82,8 \%)$ de varones. Por medio del test de proporción no se observó diferencia estadística significante en el absentismo entre los sexos $(\mathrm{p}=0,9588)$.

Tabla 1. Proporción de absentismo según el sexo

\begin{tabular}{cccc}
\hline Sexo & Certificados & Total de funcionarios & Proporción \\
\hline Femenino & 316 & 275 & 1,15 \\
Masculino & 1525 & 1105 & 1,38 \\
\hline $\mathrm{p}=0,9588$ & & \\
\hline
\end{tabular}

Se dividió la población estudiada en franjas etarias de 10 años, siendo la de 20 a 29 años la más ausente. Las razones odontológicas y médicas más indicadas en los certificados y que provocaron la ausencia del trabajador fueron dientes incluidos e impactados (CIE-10: K01) y diarrea y gastroenteritis de origen infeccioso presumible (CIE-10: A09), respectivamente. 
La mayoría de los periodos de descanso por motivos odontológicos y médicos tuvo la duración de un día, siendo que los motivos odontológicos llevaron a los trabajadores a ausentarse del trabajo por hasta el máximo de 5 días, y los médicos por 15 días.

Los individuos que se ausentaron del trabajo tenían funciones ligadas a la línea de producción (no administrativas).

En total se perdieron aproximadamente 3912 días de trabajo, de los que 83 (2,1\%) por razones odontológicas y $3829(97,9 \%)$ por razones médicas. La relación entre el número de días perdidos y el número de trabajadores (índice de gravedad) fue de 2,83; la relación entre el número de periodos de descanso médico y el número de trabajadores (índice de frecuencia) fue de 1,33 y la relación entre el número de días perdidos y el número de periodos de descanso médico (duración media de ausencias) fue de 2,12.

Tabla 2. Índice de gravedad, de frecuencia y duración media de las ausencias

\begin{tabular}{ccc}
\hline & Índice de Gravedad & \\
\hline Número de días perdidos & Número de empleados & Índice \\
3912 & 1380 & 2,83 \\
\hline & Índice de frecuencia & \\
\hline Episodios de ausencia & Número de empleados & Índice \\
1841 & 1380 & 1,33 \\
\hline & Duración media de ausencias & \\
\hline Número de días perdidos & Episodios de ausencia & Índice \\
& 18412 & 2,12 \\
\hline
\end{tabular}

\section{DISCUSIÓN}

La ocurrencia de un índice de absentismo considerado como normal en las empresas, en una profesión o en un país es discutible. En realidad, se debe tener como propósito final reducirlo el máximo posible, una vez que las enfermedades son inherentes al ser humano y lo acompañan desde sus orígenes ${ }^{12}$.

Según la legislación brasileña, el médico y el cirujano dentista son los únicos profesionales legalmente habilitados a asegurar la ausencia del trabajador y, así, garantizar el pago de su sueldo sin descuentos. Desde el punto de vista legal, se reconoce la importancia del estado mórbido de competencia odontológica como factor de absentismo por medio de la Ley 6.215, de 30 de junio de 1975, que presenta la siguiente redacción en su artículo 6. ${ }^{\circ}$, párrafo III- "Asegurar en el sector de su actividad profesional, estados mórbidos y otros, incluso para justificar las ausencias al trabajo» ${ }^{15}$. Todavía, es indispensable que el certificado presente el código de la enfermedad basado en la Clasificación Internacional de Enfermedades (CIE) ${ }^{11}$, conforme especificado en la Consolidación de las Leyes del Trabajo (CLT) y acuerdos entre empleados y empleadores (convenciones colectivas de trabajo) $)^{1,16}$.

El absentismo de corta o larga duración debe ser diferenciado. Las ausencias prolongadas se deben generalmente a enfermedades; ya las de corta duración, que son las predominantes, se pueden atribuir a causas diversas como dificultades de adaptación psicológica y condiciones sociales ${ }^{12}$. En este trabajo, se verificó que la mayoría de las ausencias fueron de corto periodo, lo que sugiere la utilización de licencia médica como forma de agresión al superior y a la institución, pues representan una forma legal que los trabajadores tienen de ausentarse del trabajo y también una válvula de escape de tensiones sociales, económicas y psicológicas. Es un punto crítico a ser afrontado en la lucha contra el absentismo ${ }^{13,17}$. 
En las personas de edad más adelantada, normalmente el absentismo presenta una menor frecuencia, sin embargo la duración de los periodos de descanso médico son mayores, al contrario de lo que ocurre con los trabajadores jóvenes ${ }^{4,12}$, lo que está de acuerdo con los hallazgos de este estudio, en el que se verificó que el ausentismo al trabajo fue mayor entre los trabajadores de franjas etarias menores.

Usualmente, las mujeres se ausentan más del trabajo que los hombres y la duración de esas ausencias es menor en las trabajadoras de edad más adelantada debido a la menor necesidad de cuidar a los hijos y hogar ${ }^{12}$. Las mujeres que trabajan fuera del hogar tienen como desafío la dupla jornada al asumir las responsabilidades inherentes a la propia actividad profesional, bien como las de la casa y de su familia. Llegan al trabajo cansadas por la fatiga residual y por lo que ya hayan hecho antes de salir de casa. Es común que la mujer se preocupe con problemas que ocurrieron o que puedan estar ocurriendo en su hogar durante el horario de trabajo y, una vez en casa, igualmente se preocupen con los problemas profesionales que la esperen el otro día ${ }^{18}$. Sin embargo, en este estudio no se observaron diferencias significativas en el absentismo entre los sexos, lo que difiere de los hallazgos de otras investigaciones ${ }^{19-21}$.

Se observó también mayor número de ausencias de trabajadores en funciones de menor remuneración, en concordancia con otros estudios ${ }^{19,21}$. Se cree que los trabajadores más calificados y que reciben mayor sueldo se ausenten menos debido a la satisfacción con su sueldo o mejor alimentación, por consecuencia, se enferman menos ${ }^{19}$.

Aunque diversos autores relatan la importancia de los problemas bucodentales en el absentismo ${ }^{6,22-24}$, se verificó que las razones odontológicas tuvieron poco peso en relación con el total de ausencias, concordando con otros estudios ${ }^{19,21,25}$. Una razón posible es que las urgencias como extracción o abscesos dentales son los únicos motivos normalmente aceptados como justificativa por las empresas ${ }^{10,21}$, difícilmente ocasionando más que un día de descanso médico; por tanto, hay una desobediencia a la Ley $6.215^{6}$, y el empleado, a pesar de conocer sus derechos, no contesta la recusa del certificado debido a la posibilidad de perder el empleo. La ausencia de la Clasificación Internacional de Enfermedades (CIE) ${ }^{11}$ en la mayoría de los certificados odontológicos también parece haber contribuido con esa ocurrencia, pues se exige su identificación para garantizar el pago del día de trabajo perdido por motivos de enfermedad. Aunque es atribución del cirujano dentista justificar la ausencia al trabajo, esta investigación enseñó que la mayor parte de eses profesionales describía, en el certificado, el acto practicado, lo que sugiere desconocimiento del sigilo profesional o de la CIE, la cual se estableció para que profesionales del área de la salud se comunicaran sin describir el tratamiento realizado, no rompiendo, así, el sigilo profesional ${ }^{26}$.

\section{OBSERVACIONES FINALES}

La preocupación principal para la dirección de la empresa sobre los problemas bucales debe ser con la caries dental, a causa de su alcance mundial, naturaleza insidiosa y acción deletérea, que puede ocasionar síndromes agudas o crónicas y generar el absentismo de cuerpo presente y, consecuentemente, accidentes laborales ${ }^{5,22}$.

Los cirujanos dentistas necesitan recibir orientaciones sobre la importancia de rellenar el certificado odontológico con el registro de la Clasificación Internacional de Enfermedades (CIE), sea para fines laborales, sea para mantener el sigilo profesional. Además, deben exigir que el certificado sea acepto, resguardando su derecho legal. Por otro lado, se debe cohibir la acción de profesionales médicos o cirujanos dentistas que, por razones menos nobles, ofrecen certificados gratuitamente, o sea, sin que el paciente presente verdaderamente el problema de salud descrito en el certificado y que justifique su ausencia en el trabajo, lo que perjudica no solo la colectividad empresarial, sino también el Sistema de Seguridad Social ${ }^{27}$. 
La clase odontológica necesita unir sus fuerzas para que, con la creación de la especialidad de la Odontología Laboral, el cirujano dentista obligatoriamente tome parte del equipo del Servicio Especializado en Ingeniería de Seguridad y en Medicina del Trabajo (SESMT), pues el examen de ingreso debería tener como criterio mínimo de exigencia la eliminación de dientes por medio de extracción y también profilaxis simple de tártaro y cálculos, contribuyendo, así, para el aumento de la productividad, seguridad laboral y disminución del absentismo?. El cirujano dentista dentro de la industria sería la única forma realista de equilibrar dos intereses: el bienestar de los trabajadores, una población usualmente excluida de levantamientos epidemiológicos y de acciones preventivas, educativas y curativas, y el desarrollo normal de la producción de la empresa ${ }^{28}$.

\section{REFERENCIAS BIBLIOGRÁFICAS}

1. Martins SP. Direito do trabalho. 15 ed. São Paulo: Atlas; 2002.

2. IOB. Segurança e saúde no trabalho. 3 ed. São Paulo: Gráfica Editora; 1993.

3. Carrion V. Comentários à consolidação das leis do trabalho. 19 ed. São Paulo: Saraiva; 1995.

4. Muñoz MM. Absentismo laboral. In: Benavides FG, Ruiz Frutos C, García García AM. Salud laboral: conceptos y técnicas para la prevención de riesgos laborales. Barcelona: Masson; 1997. p. 351-61.

5. Midorikawa ET. A odontologia em saúde do trabalhador como uma nova especialidade profissional: definição do campo de atuação e funções do cirurgião-dentista na equipe de saúde do trabalhador. 2000.337 f. Dissertação (Mestrado em Odontologia)-Faculdade de Odontologia, Universidade de São Paulo, São Paulo, 2000.

6. Borrás JS, Sanfilippo OA. Problemas odonto-estomatológicos en el trabajo. In: Martinez-Cortés F. La salud en el trabajo. México: Novum Corporativo, 1988. p. 273-9.

7. Brasil. Conselho Federal de Odontologia. Resolução CFO-25/2002 de 16 de maio de 2002. [Consultado 15 jan. 2012]. Disponible en: http://cfo.org.br/servicos-e-consultas/ato-normativo/?id=375.

8. Brasil. Ministério do Trabalho e Emprego. NR 4 - Serviços Especializados em Engenharia de Segurança e em Medicina do Trabalho. [Consultado 15 jan. 2012]. Disponible en: <http://portal.mte.gov.br/data/files/ 8A7C812D36A2800001388128376306AD/NR-04\%20\%28atualizada\%29.pdf>.

9. Medeiros EP. Exame odontológico pré-admissional. O Incisivo 1965; 4(1/2):18-22.

10. Pinto VG. Saúde Bucal: odontologia social e preventiva. 3 ed. São Paulo: Ed. Santos; 1992.

11. Organização Mundial de Saúde. CID-10: classificação estatística internacional de doenças e problemas relacionados à saúde. 5 ed. São Paulo: Edusp; 1997.

12. Forssman S. El absentismo en la industria. Boletín de la Oficina Sanitaria Panamericana 1956; 41(6):526-30.

13. Quick TC, Lapertosa JB. Análise do absentismo em usina siderúrgica. Revista Brasileira de Saúde Ocupacional 1982; 10(40):62-7.

14. Serviço Brasileiro de Apoio às Micro e Pequenas Empresas. Critérios de Classificação de Empresas: EI ME - EPP. [Consultado 15 jan. 2012]. Disponible en: <http://www.sebrae-sc.com.br/leis/default. asp?vcdtexto $=4154>$.

15. Brasil. Lei n. 6.215 de 30 de junho de 1975 - Diário Oficial da União, Brasília, DF, 1 jul. 1975. [Consultado 15 jan. 2012]. Disponible en: <http://www81.dataprev.gov.br/sislex/paginas/42/1975/6215. htm>.

16. Sindicato das Indústrias Metalúrgicas, Mecânicas e de Material Elétrico. Convenção coletiva de trabalho. Araçatuba, 2000.

17. Valtorta A, Sidi E, Bianchi SCL. Estudo do absenteísmo médico num hospital de grande porte. Revista Brasileira de Saúde Ocupacional 1985; 13(51):55-61.

18. Gomes DL. A mulher, seu trabalho e as implicações em saúde. Revista Paulista de Enfermagem 1986; 6(2):91-6.

19. Cartaxo RM. Absenteísmo em empresas industriais de Campina Grande-Paraíba: um estudo de suas causas. 1982. 132 f. Dissertação (Mestrado em Odontologia)-Centro de Ciências da Saúde, Faculdade de Odontologia, Universidade Federal do Rio Grande do Norte, Natal; 1982.

20. Danatro D. Ausentismo laboral de causa médica en una institución pública. Revista Médica del Uruguay 1997; 13(2):101-9. 
21. Martins RJ, Garbin CAS, Garbin AJÍ, Moimaz SAS. Absenteísmo por motivos odontológico e médico nos serviços público e privado. Revista Brasileira de Saúde Ocupacional 2005; 30(111):9-15.

22. Medeiros EP. Necessidades de tratamento odontológico em operários de São Paulo. O Incisivo 1970: 9(1):13-21.

23. Guzmán MF. Las enfermidades bucales como factor de pérdidas económicas en México. Revista de la Asociación Dental Mexicana 1977; 34(5):346-52.

24. Baroni G. A influência da dor de dente. Rev. CIPA 1996; 17(202):40-51, 1996.

25. Rocha JAD. Absenteísmo ao trabalho por doença e a implicação da saúde bucal como um dos seus fatores numa indústria metalúrgica da cidade de Canoas. 1981. 100 f. Dissertação (Mestrado em Odontologia)Centro de Pesquisa em Odontologia Social, Faculdade de Odontologia, Universidade Federal do Rio Grande do Norte, Natal; 1981.

26. Saliba-Garbin CA, Moimaz SAS, Saliba TA, Garbin AJI. O cirurgião-dentista e a emissão de atestados odontológicos. Odontologia e Sociedade 2000; 2(1/2):89-92.

27. Amaral AC, Róscoe JS. Causas odontológicas como absentismo na indústria. Arquivos do Centro de Estudos da Faculdade de Odontologia da Universidade Federal de Minas Gerais 1970; 7(1):127-41.

28. Pimentel OJ. Odontologia do trabalho. Odontólogo Moderno 1976; 3(2):98-9. (Carta)

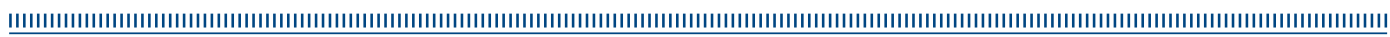

